\title{
Teaching Protein Crystallization by the Gel Acupuncture Method
}

\author{
J. M. García-Ruiz,* A. Moreno,** F. Otálora, and D. Rondón
}

Laboratorio de Estudios Cristalográficos, Instituto A ndaluz de Ciencias de la Tierra, CSIC-Universidad de Granada, Av. Fuentenueva s/ n. 18002 Granada, Spain

\section{Viedma}

Departamento de Cristalografía y Mineralogía, Facultad de Geología, Universidad Complutense Madrid 28008 Spain

\section{F. Zauscher}

Instituto de Q uímica, Universidad Industrial de Santander, Bucaramanga, Colombia

Solving the three dimensional structure of proteins by $X$-ray diffraction is an important step toward understanding their function in biological systems. The information recovered from these studies is used by research groups in chemistry and molecular biology to design new drugs and introduce specific modifications into protein molecules. In spite of the variety of crystallization methods that have been developed (1-3), a major bottleneck for this research is the production of protein single crystals with the quality and size required for X-ray structural studies. M oreover, the huge variety of proteins in biological systems will yield new crystalline materials with physical (electrical, optical, etc.) and chemical (catalysis) properties still unexplored but which will be a source for technologically advanced materials in the next century. Therefore, it seems worthwhile to teach undergraduate students in biology and chemistry how macromolecular crystals form and the techniques to grow them.

The purpose of this paper is to describe a simple method to grow protein single crystals and a set of selected experiments bringing a hands-on experience. These experiments are inexpensive and straightforward enough for teaching in any general chemistry or biochemistry laboratory. They use crystallization conditions previously tested and cover a wide range of proteins and growth behavior.

\section{Protein Solubility}

The solubility of proteins varies with several parameters, the most important being temperature and ionic strength of the solution. Since the experiments proposed are isothermal, the solubility behavior of the protein can be properly described here by using a phase ( 0 stwald-M iers) diagram as shown in Figure 1, where the equilibrium concentration (solubility) of the protein is plotted against precipitating agent concentration. Although several organic compounds and polymers have the ability to precipitate proteins (4), we will restrict the discussion to inorganic salts as precipitating agents because their effects are better known and also because they are easier to handle in teaching laboratories. As a general rule, the solubility of proteins increases with increasing ionic strength at low salt concentrations $(<0.5 \mathrm{M})$; this effect, known as "salting in", is currently explained by nonspecific electrostatic interactions between ions in the framework of the $\mathrm{D}$ ebye- $\mathrm{H}$ ückel theory

*Corresponding author. Email: jmgruiz@goliat.ugr.es.

**C urrent address: Instituto de Q uímica, Universidad $\mathrm{N}$ acional Autónoma de México.
(5). At high salt concentration, the solubility of the proteins is mainly governed by hydrophobic effects and decreases with salt concentration ("salting out"). The simplest equation that accounts for this double dependence of the solubility over the whole range of salt concentrations is $G$ reen's well-known phenomenological law (6) expressed by the relation:

$$
\log C_{e}=\log C_{0}+k_{i} \sqrt{C_{s}}-k_{0} C_{s}
$$

where $C_{e}$ is the solubility (concentration at equilibrium) of the protein, $\mathrm{C}_{0}$ is the solubility of the protein in pure water (making more algebraic than chemical sense because most proteins are unstable under these conditions), $\mathrm{C}_{\mathrm{s}}$ is the concentration of the salt used as precipitating agent, and $k_{i}$ and $\mathrm{k}_{0}$ are respectively the salting-in and salting-out constants that describe the relative importance of each effect. $N$ ote that the signs $\left(+k_{0}\right.$ and $\left.-k_{0}\right)$ show the direct and inverse relations among the solubility and salt concentrations for the salting in and salting out, respectively. Any protein solution out of equilibrium lies in the phase diagram either in the undersaturation region when $\mathrm{C}<\mathrm{C}_{\mathrm{e}}(\sigma<1)$ or in the supersaturated region when $C>C_{e}(\sigma>1)$, where $\sigma=C / C_{e}$, known as "relative supersaturation", quantitatively describes the

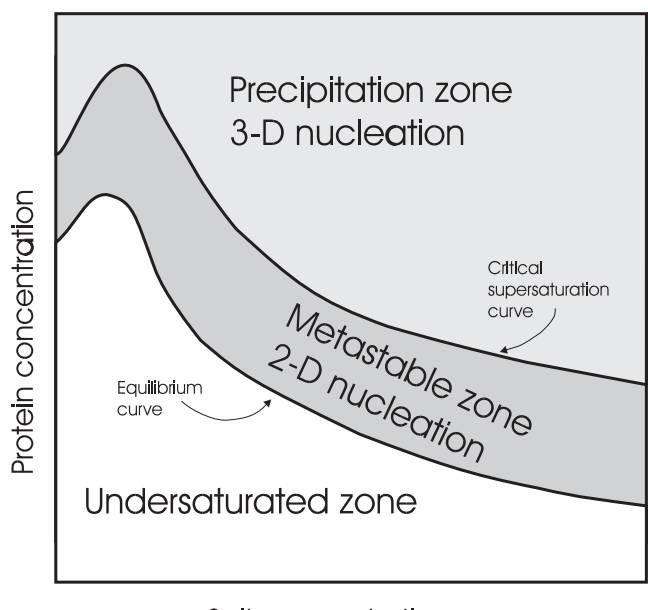

Salt concentration

Figure 1. Scheme of a phase diagram showing the variation of the equilibrium concentration (solubility) of an ideal protein as a function of the concentration of the precipitating agent. 
driving force for the separation of a solid phase containing the solute excess. This phase transition implies the overcoming of a kinetic threshold known as "critical supersaturation":

$$
\sigma^{*}=\frac{C^{*}}{C_{e}}
$$

The values taken by $C *$ for different values of the ionic strength in the above equation define a curve (sometimes called "critical supersaturation curve") that divides the supersaturated region into two other areas: the region $C>C *$, where homogeneous nucleation occurs, and the region $\mathrm{C}_{\mathrm{e}}<\mathrm{C}<\mathrm{C}^{*}$, where no spontaneous nucleation takes place but previously formed (or seeded) crystals can grow. It is important to note that, unlike the equilibrium curve, the critical supersaturation curve is kinetic in nature and therefore its location in the phase diagram depends on the supersaturation rate, a parameter largely controlled by the experimental setup. In fact, the strategy currently used to induce the crystallization of macromolecules is to bring a starting undersaturated solute-solvent system to a finite degree of supersaturation and to try to do it at a very slow rate.

We have developed a method to grow single crystals of biological macromolecules inside capillary tubes $(7,8)$. The method is very easy to implement and allows one to follow the growing process using a magnifying glass or to record it with a videocamera.

\section{Experimental Details}

The method is based upon the properties of gels, which are used to hold capillaries containing the protein solution and also to act as the mass transport medium for the precipitating agent. The experimental arrangement consists of the six steps summarized in Figure 2, which we will describe in detail.

\section{Step \#1: Preparation of the Gel Layer}

Except for the case of carboxypeptidase, all the experiments are carried out using a silica gel prepared as follows. Sodium silicate solution is commercially supplied with a density about $1.39 \mathrm{~g} / \mathrm{mL}$, which has to be lowered to $1.06 \mathrm{~g} / \mathrm{mL}$. Silica gel forms by the polymerization of the monosilicic acid, which is achieved by acidifying the sodium silicate solution. We recommend using polypropylene volumetric materials and

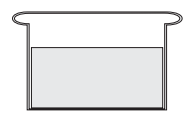

1

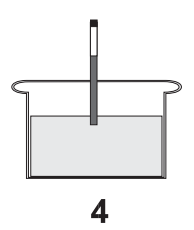

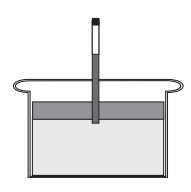

5

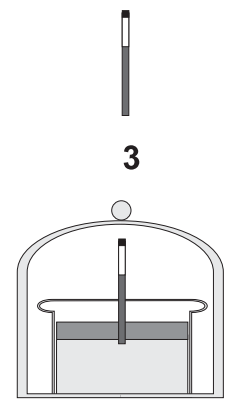

6
Figure 2. Diagrammatic representation of experimental setup for the gel acupuncture technique. The ratio between the volumes of the precipitating solution and the gel layer is one of the variables affecting the results. For simplicity we recommend a $1: 1$ ratio. freshly made sodium silicate solutions. $\mathrm{The} \mathrm{pH}$ of the gel is selected to fit the $\mathrm{pH}$ required for the crystallization of the protein, which is related to its isoelectric point. Gel pH can be selected by adding the required volume of sodium silicate solution to a $1 \mathrm{M}$ solution of acetic acid with continuous stirring. (Table 1 shows the data required to adjust to the desired value of gel $\mathrm{pH}$.) The gel is allowed to stand until complete polymerization, for a number of hours that depends on the gel $\mathrm{pH}$ (see Table 1). To inhibit bacterial growth, $0.1 \%$ (w/v) sodium azide is added to the precipitating agent solution. (CAUTION: Handle sodium azide with care because of its toxicity. This reagent can be eliminated from the preparation at the risk of having bacterial growth ruin the experiment. When using phosphate buffer, addition of sodium azide is mandatory because there is almost no chance of avoiding bacteria.)

\section{Step \#2: Filling the Capillaries with the Protein Solution}

The crystallization will take place inside capillaries. We know that the inner diameter of the capillary affects the nucleation density and therefore, for teaching purposes, it is convenient to eliminate this variable by al ways using the same type of capillary for comparative studies. We suggest the use of capillaries up to $1.2 \mathrm{~mm}$ in inner diameter and $80 \mathrm{~mm}$ in length. M elting point capillaries are suitable for optical examination of the crystallization experiments but, if this practical teaching is to be completed with some X-ray crystallographic studies, quartz capillaries should be used as growth cells to reduce subsequent handling of the crystal for mounting. It is well known that impurities modify crystallization behavior in unpredictable ways. Therefore, the capillaries must be carefully cleaned with glass detergent and well washed with double-distilled water and finally with acetone. O nce dried, they are filled with the protein solution by capillarity. Capillaries should be filled to a level a few millimeters below the upper end-avoiding wetting this end, which has to be seal ed later. It is important to use freshly made protein solutions to obtain good reproducibility.

\section{Step \#3: Sealing the Capillaries}

After the capillary is filled, its external surface remains soaked with protein solution. Clean this surface by rubbing it gently with a piece of cleaning paper. The upper part of the capillary is sealed with Plasticine or green mounting clay.

Table 1. Sodium Silicate Used To Obtain
a Specific pH Value and Gelling Time
\begin{tabular}{|ccc|}
\hline pH & $\begin{array}{c}\text { Sodium Silicate } \\
(\mathrm{mL})\end{array}$ & $\begin{array}{c}\text { G elling Time } \\
(\mathrm{h})\end{array}$ \\
\hline 4.5 & 7 & 12 \\
5.0 & 10 & 8 \\
5.5 & 12 & 6 \\
6.0 & 13 & 2 \\
6.5 & 13.5 & 1 \\
7.0 & 14 & instantaneous \\
\hline
\end{tabular}

$\mathrm{N}$ ote: These quantities of sodium silicate are calculated for material supplied by Aldrich. For other suppliers, preliminary gelling tests are recommended. The sodium silicate solution $(1.06 \mathrm{~g} / \mathrm{mL}$, $\mathrm{pH}=11.8)$ is added to $10 \mathrm{~mL}$ of $1 \mathrm{M}$ acetic acid. 
Step \#4: Puncture of the Capillaries in the $G$ el Layer

The gel is then punctured with the open end of the capillary. The suggested penetration length in the gel is $10 \pm 2 \mathrm{~mm}$. To avoid the formation of bubbles inside the capillary when punching the gel, it is desirable to have a thin liquid film on top of the gel. This film may form spontaneously by gel syneresis; otherwise it can be created by pouring a small amount of buffer solution (or double-distilled water) on the surface of the gel before puncture.

Step \#5: Pouring the Solution of the Precipitating A gent

After placing the capillaries, the precipitating solution is slowly poured on the gel. The volume of the gel, $V_{g}$, and the volume $V_{s}$ and concentration $C_{s}$ of the precipitating solution will control the final equilibrium concentration of precipitating agent inside the capillaries. All experiments here described are carried out with $\mathrm{V}_{\mathrm{s}} / \mathrm{V}_{\mathrm{g}}=1$, and therefore the final equilibrium concentration will be $\mathrm{C}_{\mathrm{s}} / 2$.

\section{Step \#6: Enclosing the System}

To avoid evaporation of the precipitating solution, the vessel with the capillary is placed onto a glass plate and enclosed inside a bigger vessel turned upside down. The lip of the second vessel should be coated with vacuum grease.

We suggest performing the laboratory work in two steps. The first step, to prepare the solutions and to set the gel layer, will take about 2-3 hours (see Table 1). The second part consists of the actual implementation of the technique (steps 26) and can be performed in 40 minutes. All the experiments described can be carried out at room temperature. After a few hours (this waiting time depends on the protein to be crystallized, see Table 2 ) it is possible to detect nucleation in
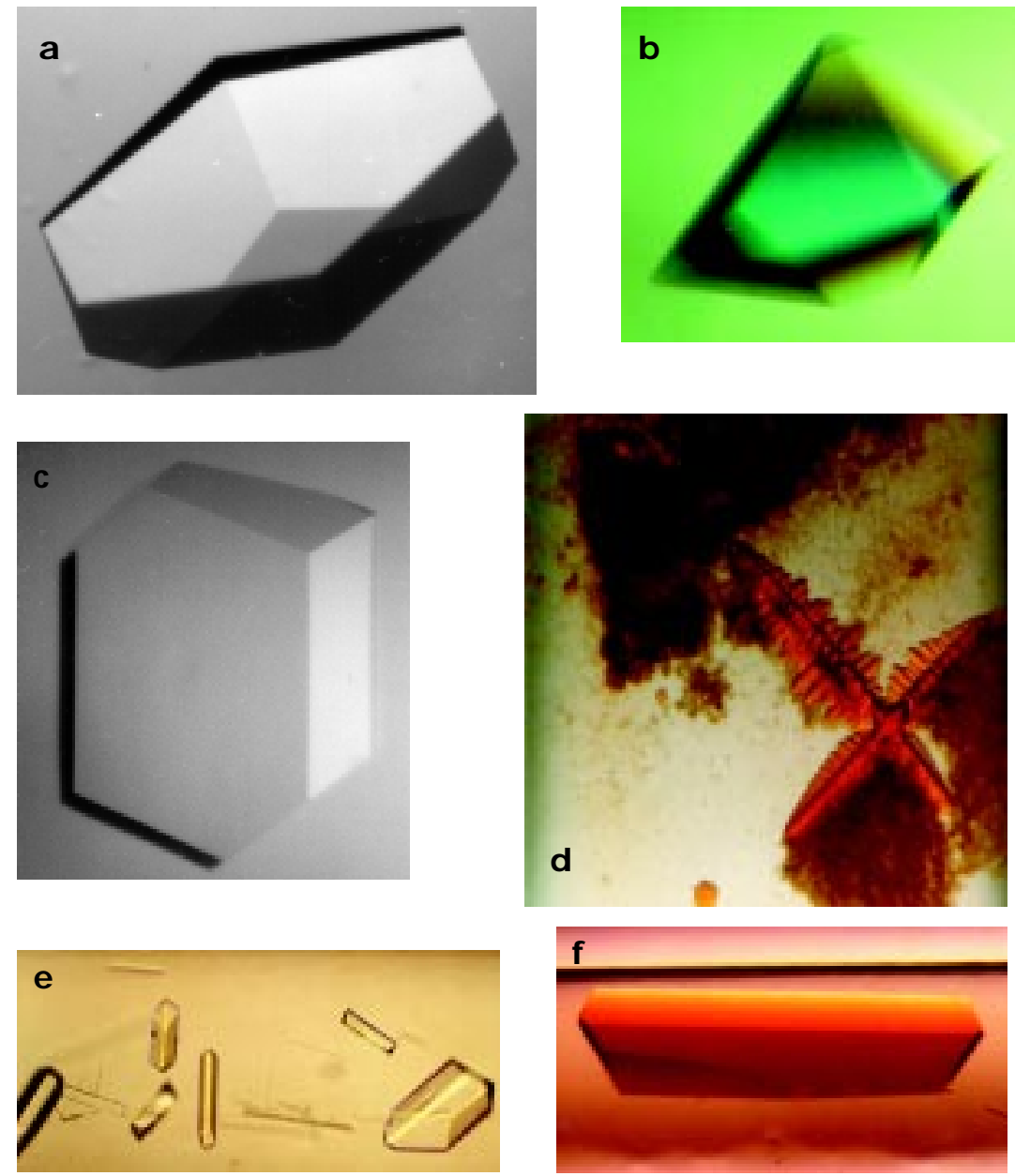

Figure 3. Protein single crystals obtained by gel acupuncture technique: (a) lysozyme; (b) thaumatin I; (c) insulin; (d) ferritin; (e) carboxypeptidase; (f) catalase.

the lower part of the capillary. Later examination can be done daily. Figure 3 shows some photomicrographs of the crystals obtained.

The molecular weight, source, price, and crystallographic system of the selected proteins are summarized in Table 3. Crystallization conditions for different types of proteins, the time needed to get crystals, and the final size of these crystals are listed in Table 2. N ote the range of molecular weights, biological sources, and crystallographic systems of this set of commercially available proteins. Finally, it should be noted that the proteins recommended for testing in this paper have reasonable costs (see Table 3 ) appropriate for consideration as a practical study case in laboratories of biochemistry or general chemistry. All glassware, including melting point capillaries, used in the experimental work is standard laboratory equipment. 
The phenomenological aspects of the technique are shown in Figure 4. The precipitating agent solution poured onto the gel layer starts to diffuse through the porous gel network. The protein solution in the capillary also diffuses through the gel, but at a much slower rate (typically, the diffusion constant of the large macromolecules is one or two orders of magnitude smaller than that of the small molecules used as precipitating agent [9]). Therefore, the salt reaches the open side of the capillary sooner than the protein molecules and starts to diffuse up into the capillary. It is important to realize that we are dealing with an open system moving toward equilibrium. As the salt diffuses up through the capillary with time, the system experiences precipitation phenomena that take place at different values of both supersaturation and rate of supersaturation. The development in time of supersaturation is rather complex (see O tálora and García-Ruiz [10] for a computer simulation of the problem), but we can highlight several features of the system. Figure 4c shows a spatiotemporal map of the variation of salt concentration inside the capillary obtained by computer simulation. N ote that such a map covers a wide range of plausible precipitation conditions, from those which are very far from equilibrium (where amorphous precipitation is expected) to those slowly approaching equilibrium (where few large and well-faceted single crystals are expected to form). By inserting in the computer simulation the appropriate parameters accounting for precipitation and dissolution, the computed maps of salt and protein concentration can be converted into a supersaturation map by using eq 1 where precipitation events are recorded (Fig. 4d).

After the short time needed to bring the precipitating agent through the gel into the capillary, the salt and protein molecules meet each other in the lower part of the capillary and the system starts to move in the phase diagram (along the trajectory $1 \rightarrow 1^{\prime}$ in Fig. 4a). The pump for the precipitating agent is the gradient $\partial \mathrm{C}_{d} \partial \mathrm{x}$, which at this time is large, and therefore the trajectory $1 \rightarrow 1^{\prime}$ is traversed quickly. For proteins (such as lysozyme) with a narrow region of salting in, the system invades the region of very high supersaturation and a large amount of protein precipitates in the form of small amorphous particles in the lower part of the capillary. The concentration of protein at this location is depleted until its equilibrium concentration (trajectory $1^{\prime} \rightarrow 2$ in Fig. 4 a) and the precipitation front working as a sink for protein molecules create a gradient of protein concentration that induces a diffusive transport. Protein concentration stays at its equilibrium value all along the region where precipitate exists. Supersaturation starts to increase again ahead of the precipitation front because of the continuous pumping of precipi-

Table 3. Properties of the Selected Proteins

\begin{tabular}{|lcllc|}
\hline Protein & MW & Source & Crystal System & $\begin{array}{c}\text { Price/ mg } \\
\text { (U.S. \$) }\end{array}$ \\
\hline Lysozyme & 14,300 & Hen egg white & Tetragonal & 3 \\
Thaumatin I & 23,000 & Thaumacoccus danielly & Hexagonal & 80 \\
Insulin & 50,000 & Porcine pancreas & Cubic & 6 \\
Concanavalin A & 102,668 & Jack bean & O rthorhombic & 0.3 \\
Carboxypeptidase & 34,472 & Bovine pancreas & Monoclinic & 1 \\
Catalase & 250,000 & Bovine liver & Orthorhombic & 15 \\
Ferritin & 456,000 & Horse spleen & Cubic (orange) & 33 \\
Apoferritin & 456,000 & Horse spleen & Cubic & 0.8 \\
\hline
\end{tabular}

tating agent from the gel (trajectory $2 \rightarrow 2^{\prime}$ ), but now this path is obviously traversed at a slower rate. This increasing supersaturation eventually gives rise to a new precipitation event, which, because of the protein concentration gradient, takes place further from the open end of the capillary at a slower supersaturation rate, leading to a polycrystalline precipitate. Protein concentration at this point falls again (trajectory $2^{\prime} \rightarrow 3$ ). The system experiences iterative phenomena of this type, yielding successively fewer and larger crystals at upper locations in the capillary as time advances. This trend is modified by two effects. The first is the finite size of the capillary- which accounts for the cessation of growth of the last-forming crystals before they reach their expected size, because protein is exhausted. The second is the dissolution of the amorphous precipitate located at the beginning of the capillary as a result of the depletion of salt concentration at that region (see concentration maximum 
around $t=2, x=1$ in Fig. 4c). This effect makes such an amorphous precipitate work as a secondary source of protein molecules for the crystals growing in the capillary and helps explain why some crystals in the middle part of the capillary grow bigger than those in the upper part, an effect that can be clearly observed in the crystal growth of lysozyme. In fact, under certain circumstances, the amazing phenomenon of a single crystal completely filling the capillary and therefore adopting its cylindrical inner shape can be also observed (11).

The selected proteins in Tables 2 and 3 demonstrate the different behaviors of crystall izing proteins. For instance, with carboxypeptidase A, we start by dissolving the protein in a water solution of $\mathrm{LiCl}$ and then counterdiffuse pure water (or a low concentration buffer solution) to decrease ionic strength. This is because the solubility of carboxypeptidase $A$, unlike that of most proteins, increases as a function of the ionic strength. Thaumatin, a protein with a sweetening power a thousand times greater than that of sucrose (12), does not yield amorphous precipitate in the lower part of the capillary as lysozyme does because of its large salting-in region and metastability region. By crystallizing ferritin and apoferritin, the student will obtain beautiful dendrites, globular and isomorphic cubic crystals, red in the first case because of the presence of iron in this important iron-storage protein (13) and colorless in the second case because of the lack of iron in the same type of protein framework. With concanavalin $A$, the effect of polyethylene glycol as a precipitating agent can be demonstrated.

\section{Acknowledgments}

Wethank $\mathrm{G}$ eorge $\mathrm{D}$ eT itta, from $\mathrm{H}$ auptman-Woodward $M$ edical Research Institute at Buffalo, and an anonymous ref- eree for their review and criticism of the manuscript. Weacknowledge financial support from the D irección General de Investigación Científica y Tecnológica of Spain through project PB92-0056 and from CSIC. A.M., F.Z., and D.R. gratefully acknowledge grants from the $M$ inisterio de Educación y Ciencia of Spain, and A.M also acknowledges a grant from the CONACYT (M éxico).

\section{Literature Cited}

1. M cPherson, A. Preparation and Analysis of Protein Crystals; Krieger: M alabar, FL, 1989.

2. Crystallisation of N ucleic acids and Proteins: A Practical Approach; D ucruix, A.; Giegé, R., Eds.; IRL: O xford, 1992.

3. Blundell, T. L.; Johnson, L. N . Protein Crystallography; Academic: N ew York, 1976.

4. Gilliland, G. J. Crystal Growth 1988, 90, 51-59.

5. Ducruix, F. A.; Riès-Kautt, M. M ethods: A Companion to M ethodsin Enzymology 1990, 1(1), 25-30.

6. Green, A. A. J. Biol. Chem. 1932, 95, 47.

7. G arcía-Ruiz, J. M .; M oreno, A.; V iedma, C.; Coll, M. M ater. Res Bull. 1993, 28, 541-547.

8. García-Ruiz, J. M.; M oreno, A. Acta Crystallogr., Sect. D 1994, $50,484-490$

9. Robert, M. C.; Lefaucheux, F. J. Crystal Growth 1988, 90, 358-367.

10. O tálora, F.; García-Ruiz, J. M. J. Crystal Growth 1996, 169, 361-367.

11. García-Ruiz, J. M .; M oreno, A.; Parraga, A.; Coll, M. Acta Crystallogr., Sect. D 1995, 51, 278-281.

12. de Vos, A. M.; Hatada, M.; van der Wel, H.; Krabbendam, H .; Peerdeman, A. F.; Kim, S.-H. Proc. N atl. Acad. Sci. U SA 1985, 82, 1406-1409.

13. Theil, E. C.; Raymond, K. N . In Bioinorganic Chemistry; Bertini, I.; Gray, H. B.; Lippard, S.; Valentine, J. , Eds.; University Science Books: Chicago, 1994; pp 1-35. 